\title{
Nur die heutige Fortbildung gibt dem Arzt Kredit
}

Max Konzelmann

Korrespondenz:

Dr. med. Max Konzelmann Asylstrasse 39

CH-8750 Glarus

max.konzelmann@yahoo.de
Der verantwortungsbewusste Arzt, der sich zum Spezialisten weitergebildet hat, bleibt dank kontinuierlicher Fortbildung immer auf der Höhe des beruflichen Wissens. Mit Begeisterung befolgt er die einheimische Fortbildungsordnung, hält sich durch Surfen in Büchern, Zeitschriften und Internet auf dem Laufenden und erwirbt alle 3 Jahre mindestens 150 Fortbildungscredits.

Im Gegensatz zum trockenen Literaturstudium kann er für die Beschaffung der Credits von zu Hause fort, um sich zu bilden, und hat dabei Gelegenheit, Kollegen und andere Örtlichkeiten zu sehen oder kennenzulernen. Dadurch wird nicht nur sein fachmedizinischer, sondern auch der globale Horizont erweitert, und der Facharzt bleibt für die Tätigkeit am Patienten kreditwürdig.

Vor Jahrzehnten war die Fortbildung noch nicht geregelt. Es war die Zeit des largen Individualismus, der autistisch undisziplinierten Selbsteinschätzung der Fachärzte. Der Patient wusste nicht, ob er an einen Könner, an einen Jünger des Mittelmasses oder an einen jovialen Ignoranten geraten würde. Die Qualität war überhaupt nicht garantiert, und nicht selten wurstelte sich ein Arzt einfach so durch.

Zum Glück hat sich das radikal geändert. Dank der weisen Massnahmen gesundheitspolitischer Vordenker ist die Qualität des Arztes dadurch garantiert, dass er die vorgeschriebene Anzahl Credits erwirbt. Dabei ist es irrelevant, ob die Fortbildungsmaterie bis ins Bewusstsein vordringt und gespeichert wird. Verlangt wird einzig, dass man sich ihr im Hörsaal aussetzt. Der so konditionierte Arzt erhält die Legitimation zur Behandlung und wird von den Krankenversicherern als Leistungserbringer anerkannt.

Es ist nun aber so, dass die zahlreichen Fortbildungsveranstaltungen mit vielfältigen und anspruchsvollen Inhalten anstrengend sind. Aus diesem Grund ist man dazu übergegangen, parallel zur Wissenschaft auch Erholung in Form von Kongressreisen, Theaterund Konzertbesuchen, Kreuzfahrten, Skiweekends usw. anzubieten, denn nur der ausgeruhte, aufgestellte Arzt hat glücklichere Patienten.

Es liegt in der Natur der Fortbildung, dass sie über das vorhandene Wissen hinausführen muss. Sie kann ihre Funktion nicht erfüllen, wenn immer nur Bisheriges wiederholt wird. Die Programmgestalter bemühen sich daher unablässig um neue attraktive und innovative Themen. Aus ihrer Vielfalt kann dann der wissens- begierige Spezialist die ihm besonders zusagenden auswählen, um sich die nötigen Credits anzueignen.

Im Folgenden soll paradigmatisch aufgezeigt werden, wie sich eine pflichtbewusste Kollegin die noch fehlenden Credits erwirbt.

$\mathrm{M}^{\text {me }}$ Céline Lapeau, die Hautärztin in Bellelaye, erschrak, als sie im Sommer den Bestand ihrer Fortbildungscredits kontrollierte. Sie weiss, dass sie den Besitz von 150 Punkten nachweisen muss, wenn die Fortbildungsprotokollstichprobe auf ihren Namen trifft. Aber auch mit zweimaligem Nachzählen kommt sie nur auf 112 Credits.

Es müssen also noch 38 beschafft werden. Ohne diese ist sie nicht fähig, ihre Arbeit als Dermatologin zum Wohle der Patienten auszuführen, und muss auch mit Sanktionen bis hin zum Berufsverbot rechnen.

Fieberhaft blättert sie die vielfältigen Fortbildungsangebote durch und beginnt, ihr Fortbildungsprogramm zusammenzustellen:

- 3 Credits winken für den Besuch des Berner Fortbildungsnachmittags mit dem Thema «Die Bedeutung des toll-like-Rezeptors für das REM-Syndrom und seine Beziehung zur Skabiesmilbe».

- Aus Genf kann sie 3 Credits für den Besuch des Aknesymposiums «L'acné du nourrisson, réaction adverse à l'Ovomaltine?» mitnehmen.

- Weitere 8 Credits winken für die Teilnahme am La-Roche-Posay-Weekend in Gstaad mit dem Hauptthema «Managing und Entfaltung der Gesichtshaut im Umfeld ökologischer Belastung und psychotherapeutischer Verformung» (mit 6-GangDinner und Spa).

- Wenn sie sich noch rechtzeitig für Berlin anmeldet, kann sie mit weiteren 8 Credits rechnen. Dort tagt in 2 Wochen die Deutsche Arbeitsgemeinschaft zur Förderung sexuell übertragbarer Krankheiten (DAGFSÜK). Thema: «Muss die Dermatologie morgen ohne Venerea auskommen? Was können wir dagegen tun?» Ergebnisse der seit 2005 laufenden doppelblinden Feldstudie zur Reintegration der Geschlechtskrankheiten in den Europäischen Alltag (mit praktischen Beispielen, Besuch des Reichstags und Bootsfahrt auf der Spree).

- Interessant ist auch der Basler Fortbildungstag «Brushing up your knowledge in antimycotic kryotherapy» mit Vergabe von 6 Credits.

- Und das Zürcher Haarsymposium «Trübe Aussichten für die Therapie des globalen Haarsterbens. 
Bringen Stammzellen die Wende?» wirft weitere 6 Credits ab.

- Frau Dr. Lapeau ist jetzt zuversichtlich. Sie braucht nur noch 10 weitere Credits. Wenn sie die Reise nach Verona bucht, wo das Thema «La connection entre SIDA et AIDA, spéculation ou vérité?» global beleuchtet wird und 8 Credits verspricht, fehlen nur noch zwei.

- Diese zwei letzten Credits wird sie bekommen, wenn sie in Bulle am Dermo-Brunch de Gruyère über «Bullöse Dermatosen mit hohen IgE-Titern gegen Greyerzer Käse» teilnimmt.
Jetzt kann sie wieder aufatmen. Sie hat die Sammlung ihrer Credits komplettiert. Allerdings musste sie dafür ihre Sprechstunden immer wieder einmal ausfallen lassen. Aber die Patienten werden es ihr nicht verargen, denn sie haben jetzt die Gewissheit, dass Frau Doktor auch weiterhin über die Kompetenz verfügt, sie richtig zu behandeln.

\section{Literatur}

1 Schöni MH. Anmerkungen zum Fortbildungs(schwach)sinn. Schweiz Ärztezeitung. 2009; 90(11):442-3

\section{Sozialversicherungsrecht}

Myriam Schwendener

Krankheit und Recht. Der Krankheitsbegriff im schweizerischen Sozialversicherungsrecht

Basel/Genf/München: Helbing Lichtenhahn; 2008

ISBN: 978-3-7190-2811-4

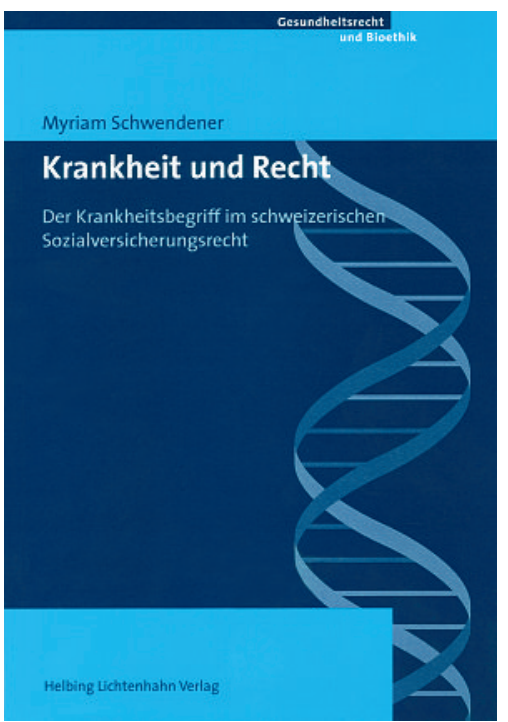

Dieses Buch stellt den Krankheitsbegriff im schweizerischen Sozialversicherungsrecht ins Zentrum der Betrachtungen und gewährt dem Leser interessante Ein- und Ausblicke in das Spannungsfeld von Medizin, Recht und Gesellschaft.
Damit der sozialversicherungsrechtliche Krankheitsbegriff seine Funktion als Definitionsgrundlage für mögliche Leistungsansprüche wahrnehmen kann, bedarf er eines Krankheitsverständnisses und einer Vorstellung von Gesundheit. Im ersten Teil des Werks wird deshalb zunächst das Begriffspaar Gesundheit und Krankheit unter medizinischen, philosophischen und soziologischen Aspekten betrachtet. Sodann werden verschiedene Gesundheits- und Krankheitskonzepte vorgestellt und analysiert, die den Wandel vom biomedizinischen zum biopsychosozialen Krankheitsmodell aufzeigen.

Auf dieser Grundlage wird anschliessend die rechtliche Dimension des sozialversicherungsrechtlichen Krankheitsbegriffs präzise ausgelotet und seine Bedeutung und Funktion aufgezeigt. Dabei wird auf das unterschiedliche Verständnis von Medizin und Recht hingewiesen und konstruktiv kritisiert: Während die Medizin dem biopsychosozialen Modell folgt, sind gemäss herrschender Rechtsprechung soziale Faktoren bei der Beurteilung einer Gesundheitsschädigung als Grundlage verschiedener sozialversicherungsrechtlicher Leistungsansprüche (Heilbehandlung oder Invalidenrenten) gänzlich auszuklammern.

Auf die rechtliche Analyse folgen Ausführungen zu praxisrelevanten Problemen in der Rechtsanwendung, die sich vorwiegend aus den unterschiedlichen Zugängen zur Thematik durch das Recht, die Medizin und die sich durch bestimmte soziale Werthaltungen auszeichnende Gesellschaft ergeben. Die unterschiedliche Denk- und Arbeitsweise und die Aufgaben der jeweiligen Akteure werden in diesem Zusammenhang erläutert, wobei der Zusammenarbeit von Arzt und Jurist eine grosse Bedeutung beigemessen wird. Die Problematik um die nicht objektivierbaren Gesundheitsbeeinträchtigungen und der Beweis (medizinischer) Tatsachen stellen vor diesem Hintergrund einen weiteren Untersuchungsgegenstand dar. Mit dem Fokus auf die Interinstitutionelle Zusammenarbeit und die neu eingeführten Massnahmen der frühzeitigen Förderung der Eingliederung im Rahmen der 5. IVG-Revision wird ein Ausblick gegeben, wie eine optimierte Gesundheitsförderung aussehen könnte und sollte.

Die Autorin analysiert die Problematik um den sozialversicherungsrechtlichen Krankheitsbegriff scharfsinnig und aus einer vielschichtigen Optik, zeigt mögliche Perspektiven auf und postuliert schliesslich die ganzheitliche Erfassung eines Patienten in seiner oft komplexen Mehrfachproblematik und damit den adäquaten Umgang der Gesellschaft mit kranken Menschen. Die Lektüre dieser kurzweiligen und sehr dichten Ausführungen ist deshalb für interessierte «Spezialisten» wie «Laien» aus beiden Fachbereichen - Recht und Medizin - gleichermassen zu empfehlen.

lic. iur. Eva Siki, Zürich 